\title{
Receptive field remodeling induced by skin stimulation in cerebellar neurons in vivo
}

\section{Henrik Jörntell* and Carl-Fredrik Ekerot}

Department of Experimental Medical Sciences, Section for Neuroscience, Lund, Sweden

\section{Edited by:}

Michael Brecht, Humboldt University

Berlin, Germany

Reviewed by:

Randy M. Bruno, Columbia University, USA

Paul Dean, University of Sheffield, UK

\section{*Correspondence:}

Henrik Jörntell, Department of

Experimental Medical Sciences,

Section for Neuroscience, BMC F10,

Tornavägen 10, SE-221 84 Lund,

Sweden.

e-mail: henrik.jorntell@med.lu.se
The receptive field of a neuron reflects its function. For example, for parallel fiber (PF) inputs in C3 zone the cerebellar cortex, the excitatory and inhibitory receptive fields of a Purkinje cell (PC) have different locations, and each location has a specific relationship to the location of the climbing fiber (CF) receptive field of the PC. Previous studies have shown that this pattern of input connectivity to the $\mathrm{PC}$ and its afferent inhibitory interneurons can be fundamentally disrupted by applying direct electrical stimulation to the PFs, paired or unpaired with CF activation, with protocols that induce plasticity in these synapses. However, afferent fiber stimulation, which is typically used in experimental studies of plasticity, set up highly artificial input patterns at the level of the recipient cells, raising the issue that these forms of plasticity potentially may not occur under more natural input patterns. Here we used skin stimulation to set up spatiotemporally more realistic afferent input patterns in the PFs to investigate whether these input patterns are also capable of inducing synaptic plasticity using similar protocols that have previously been described for direct PF stimulation. We find that receptive field components can be added to and removed from PCs and interneurons following brief periods of skin stimulation. Following these protocols, the receptive fields of mossy fibers were unchanged. These findings confirm that previously described plasticity protocols may have a functional role also for more normal patterns of afferent input.

Keywords: parallel fibers, Purkinje cells, interneurons, climbing fibers, synaptic plasticity

\section{INTRODUCTION}

The efficacy of a synaptic contact is typically subjected to bidirectional plasticity, i.e., it can be both potentiated and depressed (Malenka and Bear, 2004). The factors that determine the polarity of synaptic plasticity are dependent on the activity state of the postsynaptic cell and can in many cases be demonstrated to be dependent on the intracellular $\mathrm{Ca}^{2+}$ levels (reviewed by Jorntell and Hansel, 2006). In the cerebellum, the climbing fibers (CF) have a major role in raising the $\mathrm{Ca}^{2+}$-levels in PCs (Miyakawa et al., 1992). Consequently, PF synapses that are activated in conjunction with the climbing fiber in a PC will be depressed, leading to climbing fiber-induced PF-LTD (Ito et al., 1982). PF-LTD has been implicated in the adaptation of the vestibulo-ocular reflex (Ito, 1982, 1984, 2001, 2006), and for a long time this has been one of the clearest explanatory models of how synaptic plasticity can lead to behavioral change.

Recent in vitro and in vivo studies, using combinations of PF and $\mathrm{CF}$ activation, have in many ways changed the prerequisites for how the circuitry/synaptic mechanisms of cerebellar learning can be interpreted. First of all, PF-LTP that lead to a reversal of the CF induced PF-LTD in PCs was demonstrated in vitro (Lev-Ram et al., 2002, 2003; Coesmans et al., 2004). In vivo, PF activation alone, in a protocol that mimicked a PF-LTD protocol but omitted the $\mathrm{CF}$ activation, led to spectacular receptive field increases in PCs (Jorntell and Ekerot, 2002). Secondly, a CF input to interneurons and its mode of activation was demonstrated (Jorntell and Ekerot, 2002, 2003; Szapiro and Barbour, 2007). A CF-dependent signal in interneurons was predicted from the fact that the PF receptive field of interneurons overlapped that of the local CF (Ekerot and Jorntell, 2001), and also from the observation that CF activation converted a PF-stimulation protocol that induced receptive field reduction to a protocol that induced to receptive field expansion (Jorntell and Ekerot, 2002, 2003), most likely corresponding to LTD and LTP, respectively, of PF input to interneurons (Rancillac and Crepel, 2004; Smith and Otis, 2005; Dean et al., 2010). Due to a lack of recordings, a picture of the potential functional roles of the interneurons in vivo has only recently emerged (Jorntell et al., 2010).

However, all of the above studies involved mainly direct electrical PF stimulation, an artificial form of PF activation, which would be expected to result in highly unphysiological PF activity patterns. For example, in (Jorntell and Ekerot, 2002) burst stimulation was applied to a bundle of PFs which carried information from a multitude of receptive fields, which combined covered more or less the entire body skin. If a local PF bundle activation recruits inputs from the entire body skin, then we can expect that PFs carrying different information lie intermixed. But strong skin activation of the entire body skin in near perfect synchrony is a highly nonrealistic scenario under behavior. Rather, because of the redundancy and dense multiplication of mossy fiber inputs from a single skin area along the rostrocaudal axis of the cerebellar cortex (Garwicz et al., 1998), it is likely that PF inputs from a single skin area are distributed and represented many times in the population of PFs, representing numerous bundles, that innervate the dendritic trees of the PCs and their afferent interneurons. Therefore, in order to evaluate whether the previously described forms of plasticity can have a role in brain circuitry plasticity in vivo, we here apply intense 
but brief skin stimulation that is paired or unpaired with CFs in order to investigate the effect on the receptive fields of PCs and interneurons in vivo.

\section{MATERIALS AND METHODS}

Adult cats were prepared as previously described (Ekerot and Jorntell, 2001; Jorntell and Ekerot, 2002, 2003). Briefly, following an initial anesthesia with propofol (Diprivan ${ }^{\circledR}$ Zeneca Ltd, Macclesfield, Cheshire, UK), the animals were decerebrated at the intercollicular level and mounted in a stereotaxic frame. The animals were artificially ventilated and the end-expiratory $\mathrm{CO}_{2}$, blood pressure, and rectal temperature were continuously monitored and maintained within physiological limits. Drainage of cerebrospinal fluid, pneumothorax, and clamping the spinal processes of a few cervical and lumbar vertebral bodies served to increase the mechanical stability of the preparation. Our EEG recordings were characterized by a background of periodic $1-4 \mathrm{~Hz}$ oscillatory activity, periodically interrupted by large-amplitude $7-14 \mathrm{~Hz}$ spindle oscillations lasting for $0.5 \mathrm{~s}$ or more. These forms of EEG activities are normally associated with deep stages of sleep (cf. Niedermayer and Lopes da Silva, 1993). The pattern of EEG activity and the blood pressure remained stable throughout experiments and was unaffected by noxious stimulation.

\section{RECORDINGS AND STIMULATION}

The initial delineation of the forelimb area of the $\mathrm{C} 3$ zone in the cerebellar anterior lobe and the continuous monitoring of the general condition in the sensitive mossy fiber-to-granule cell-to-parallel fiber pathway were performed as described previously (Ekerot and Jorntell, 2001; Jorntell and Ekerot, 2002; Bengtsson and Jorntell, 2007). Also the general recording procedures and the procedures for placing stimulation electrodes in the inferior olive (IO) and among the superficial parallel fibers (PFs) have been described in detail elsewhere (Jorntell and Ekerot, 2003). For stimulation of PFs and the IO we used tungsten-in-glass microelectrodes insulated except for the last 50-120 $\mu \mathrm{m}$.

Unitary extracellular recordings from single Purkinje cells (PCs) and interneurons were made by tungsten-in-glass microelectrodes, insulated except for the last 10-30 $\mu \mathrm{m}$, in the PCs layer and in the molecular layer, respectively. All recordings were made in the superficial part of the cortex. Once a unitary recording was established, the electrode signal was continuously digitized and recorded at 50-200 kHz (Data translation A/D-board DT3010, driven by home-made software on a PC). The driving of the recorded spike from the body surface was investigated for several circumscribed skin sites on the ipsilateral body skin. To quantify the inputs, a small strain gage device was mounted on the fingertip of the investigator. Stimulation consisted in brief, short strokes applied to the skin. The signal from the strain gage indicated the onset of the stimulation, so that peristimulus histograms of the spike activity, evoked by 30-100 stimulations on each skin site, could be constructed in the off-line analysis. The activity in the first 50-100 ms after the stimulation was counted as evoked, and after subtraction of the prestimulus baseline activity (averaged over 300-400 ms) the evoked response was expressed as a ratio of the baseline activity. The net integrated strain gage signal was calculated according to the same principle. The evoked response ratio was color coded in a contour plot created by the Surfer ${ }^{\odot}$ software (Golden Software). Specific skin sites were given $X-Y$ coordinates depending on their position in an outline of the body skin, and the data fed into the contour plots was the quantified input from the specific skin sites. Histograms of spike responses evoked by inferior olivary (IO) stimulation were obtained from 15 to 60 stimulations, no baseline activity removed.

For statistical analysis, we classified the skin sites explored by manual skin stimulation into V categories, I-V. Category I skin sites corresponds to the original receptive field of the neuron recorded from. Category II is defined as skin sites that were adjacent to the original receptive field. Category III is the forelimb skin site to which the skin burst stimulation protocol was applied. Category IV corresponds to all other skin sites on the ipsilateral forelimb. Category V corresponds to all other skin sites on the body, i.e., hindlimb and trunk. Category VI corresponds to the location of the climbing fiber receptive field (only applies to PCs since the original receptive field equals the climbing fiber receptive field in interneurons (Jorntell and Ekerot, 2003).

\section{STIMULATION PROTOCOLS}

Stimulation protocols were based on the protocols of a previous study (Jorntell and Ekerot, 2002) and were named after the effect they were expected to have. Burst stimulations were applied either to the PFs or the skin. For PF burst stimulation we used intensities of $10-50 \mu \mathrm{A}$, with shocks $0.2 \mathrm{~ms}$ long. Shocks were delivered at $100 \mathrm{~Hz}$ for $100 \mathrm{~ms}$, a train that was repeated at $0.33 \mathrm{~Hz}$ for $10 \mathrm{~min}$. For skin burst stimulation we used pairs of percutaneous needle electrodes separated by $4-8 \mathrm{~mm}$ and stimulated at $1.2 \mathrm{~mA}$, with shocks $0.1 \mathrm{~ms}$ long. Shocks were delivered at $333 \mathrm{~Hz}$ for $150 \mathrm{~ms}$, and this train was repeated at $1 \mathrm{~Hz}$ for $5 \mathrm{~min}$.

Bursts were paired or unpaired with IO stimulation at $25-100 \mu \mathrm{A}$ with a single shock with a duration of $0.2 \mathrm{~ms}$. When paired with skin stimulation, the IO stimulation was delayed by $10 \mathrm{~ms}$ in order to let the climbing fiber activation occur just after the onset of skin activated PF activity (as judged by the onset latency time of the N3 field potential). Depending on the type of neuron recorded from, labels for PF burst protocols paired with IO stimulation were PC-PFLTD or interneuron-PFLTP, PF burst protocols without IO, PC-PFLTP, or interneuron-PFLTD; skin burst protocols paired with IO, PC-skinLTD or interneuron-skinLTP; and skin burst protocols unpaired with IO, PC-skinLTP or interneuron-skinLTD.

All experimental procedures confirmed to the regulatory standards and were approved in advance by the local Swedish Animal Research Ethics Committee.

\section{RESULTS RECEPTIVE FIELD PLASTICITY INDUCED BY SKIN STIMULATION IN INTERNEURONS}

All recordings were made in the forelimb area of the $\mathrm{C} 3$ zone in the cerebellar cortex (see Ekerot and Jorntell, 2003; Apps and Garwicz, 2005; Jorntell et al., 2010 for reviews). All interneuron recordings were made in the molecular layer in the superficial part of the cortex accessible from the surface (recording depths: 40-290 $\mu \mathrm{m}$ ). Interneurons were identified by having large extracellular spikes in the molecular layer (Figure 1A; which PCs lack) and by their lack of complex spikes (Csp) both spontaneously and in responses evoked 

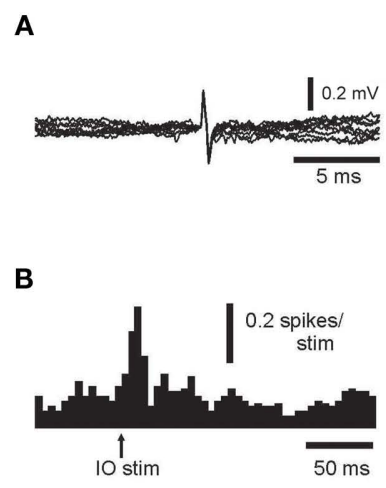

D
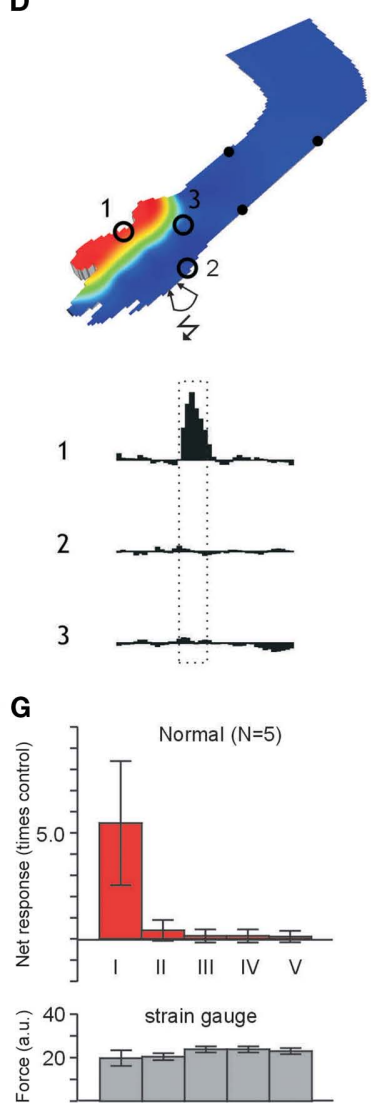

FIGURE 1 | Recruitment of input from specific skin sites to interneurons. (A) Superimposed spontaneous spikes. Scale bars: $0.2 \mathrm{mV}$ and $5 \mathrm{~ms}$, respectively. (B) Spike responses to stimulation in the $I O$ at $50 \mu \mathrm{A}$. Bin width $5 \mathrm{~ms}$. (C) Raw traces of spike responses evoked by manual stimulation at skin sites \#1 and \#2, before and after a interneuron-skinLTP protocol applied to site\#2. The corresponding strain gage signals for skin contact are shown superimposed below each data record set. (D) Quantified skin input to an interneuron under "naïve," normal conditions. Stimulated skin sites are indicated by spots. The input from skin sites indicated by numbered circles are illustrated in peristimulus histograms below. Peristimulus histograms show the net spike activity, i.e., the prestimulus baseline activity is subtracted. (histograms show -300 to +500 ms relative to the onset of the stimulation with a $20 \mathrm{~ms}$ bin width). Dashed box indicate the time of stimulation. The quantified input from these and the other stimulated skin sites is expressed as ratios of the baseline activity in the color coded contour plot. (E) Quantified skin input to the same
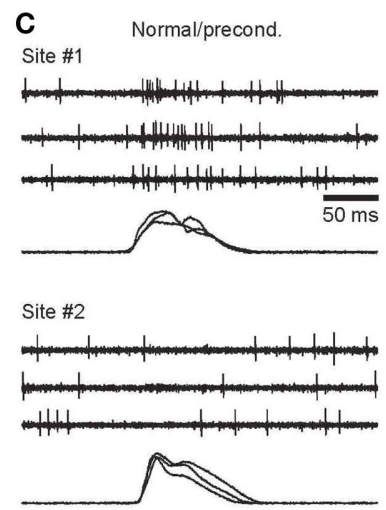

E
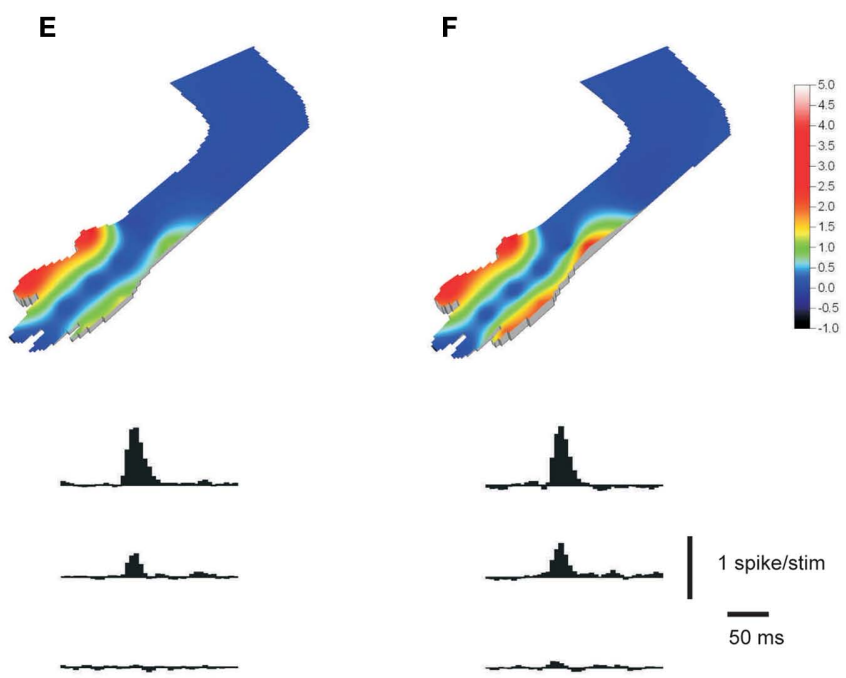

$\mathbf{H}$
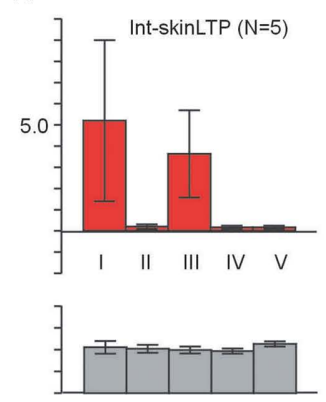

interneuron, but 10 min after the termination of an interneuron-skinLTP protocol applied to skin site \#2. Histograms illustrate input from the same skin sites as in (A). (F) Display as in (E), but with responses recorded 20 min later. Note the stronger input from site \#2. Scale bars apply to (D-F). (G) Graph summarizing the responses evoked from differently located skin sites in normal,

unconditioned interneurons. Inputs were quantified and expressed as multiples of the baseline activity. Categories of skin sites: I, original receptive field; II, skin sites adjacent to the original receptive field; III, forelimb skin site to which the interneuron-skinLTP protocol was applied; IV, other skin sites on the ipsilateral forelimb; $\mathrm{V}$, other skin sites on the body. Bottom graph, corresponding data for the strain gage signal. Data from five interneurons as indicated. All data in this and in similar displays throughout the paper are indicated by means (bars) \pm SD (capped lines). (H) Display as in (G), but for recordings 20-40 min after the termination of an interneuron-skinLTP protocol. Data from five interneurons as indicated. 
from the IO. Spontaneous spike firing frequencies of interneurons were also substantially lower than those of PCs $(14.7 \pm 8.3 \mathrm{~Hz} ; N=10$ interneurons) compared to $(37.8 \pm 3.8 \mathrm{~Hz} ; N=12 \mathrm{PCs})$ mean $\pm \mathrm{SD}$.

In normal, unconditioned interneurons, manual skin stimulation evoked spike discharges from one skin area only (Figures 1C,D,G). To quantify this observation, we used repeated skin stimulation to a number of different skin sites and made peristimulus histograms for the spike responses of each stimulated skin site (Figures 1C,D,G). Even though we used only light touch, the responses evoked from within the receptive field exceeded the baseline activity by about $500 \%$ (category I in Figure 1G). Other skin areas were classified as adjacent (skin areas lying close to the border of the receptive field, category II), other forelimb sites (4-12 different sites, category IV), and other skin sites (category V, typically 2-3 hindlimb sites and 1-2 sites on the back and belly). Stimulation of these skin sites evoked no or very little spike discharge (Figure 1G). In order to provide a control of the amount of skin stimulation applied, we also calculated the net integrated strain gage signal for each site. This signal was similar for all stimulation sites (Figure 1G, bottom graphs).

In order to change the receptive field of an interneuron, we first established that the electrode placed in the IO evoked field potentials in the recording area and spikes in the interneuron recorded from (see Figure 1B). In the example shown in Figure 1, we then used closely spaced percutaneous needle electrodes to deliver high frequency train stimulation to a localized skin site (site \#2). This stimulation was paired with a single shock to the IO, which was delayed by 8-10 ms to compensate for the conduction time from the periphery and thereby making the climbing fiber activation coincide with the earliest part of the PF discharge (cf. Jorntell and Ekerot, 2003) set up by the skin stimulation (interneuron-skinLTP protocol). During the course of about $30 \mathrm{~min}$ after termination of the stimulation protocol, a distinct input from the stimulated skin site developed (Figures 1C,E,F). This input was nearly as strong as the input from the original receptive field (360 $\pm 230 \%$ compared to $540 \pm 370 \%$ higher than baseline activity, Figure $1 \mathbf{H})$. The input from other skin areas was unchanged (Figure 1H). Also the amount of skin stimulation applied was similar for different skin sites, and also similar to the control situation (Figures 1G,H, bottom graphs).

Our previous data also indicated that PF synapses on an interneuron activated without simultaneous climbing fiber input should be depressed (Jorntell and Ekerot, 2002). This was tested for two cells recorded after new receptive field components had been added by the interneuron-skinLTP protocol. After stimulating the same skin site that was used to potentiate the skin input, but this time omitting the climbing fiber activation, the input from that skin area was nearly completely removed (remaining input $+23 \%$ and $+9 \%$ relative to the baseline activity, compared to $+520 \%$ and $+230 \%$ before).

In order to further test the depression of PF input in interneurons following skin stimulation, we first expanded the existing receptive field by using direct $\mathrm{PF}$ stimulation in bursts combined with climbing fiber activation (interneuron-PFLTP, Figure 2A; Jorntell and Ekerot, 2002). In the expanded receptive field, stimulation of a skin site, again with the same protocol as for interneuron-skinLTP but omitting the climbing fiber activation (thus an interneuron-skinLTD protocol), resulted in a selective depression in input from that part of the receptive field (input $-9 \pm 15 \%$ of the prestimulus baseline, compared to +200 to $+600 \%$, on average, from non-stimulated skin areas; Figure 2B). A depression of the input from some category IV sites (Figure 2B, upper graph to the far right) in the pooled data could be explained by that some skin sites in this category was actually localized adjacently to the category III sites, to which the interneuron-skinLTD protocol was applied. Since some mossy fibers/granule cells carry input from receptive fields that would span both these skin areas (Garwicz et al., 1998; Jorntell and Ekerot, 2006), and therefore to be activated by the skin burst stimulation, input from these granule cells would be expected to be depressed.

\section{RECEPTIVE FIELD PLASTICITY INDUCED BY SKIN STIMULATION IN PCS}

Purkinje cells were recorded at a depth of 300-350 $\mu \mathrm{m}$ from the cortical surface. PCs were identified by the simultaneous recording of simple spikes (Ssp) and Csp (Figure 3A). Csp represent activation of the PC by CF from cells in the IO. Direct electrical stimulation in this nucleus consequently drives the Csp (Figure 3A). Ssp was not driven by olivary stimulation, which serves as an additional distinguishing criterion relative to the interneurons (cf. Figures $3 \mathrm{~A}$ and $1 B$ ). In the account of evoked responses below, Ssp were distinguished from Csp but only simple spike activation is considered. Examples of spike responses are shown in Figure 3B, in which the input and strain gage signals on stimulation of skin site \#3 are compared before and after conditioning with a PC-PFLTP protocol.

The PF receptive fields of normal PCs differ fundamentally from those of interneurons in that they are located strictly outside the climbing fiber receptive field (Ekerot and Jorntell, 2001, 2003). However, also in PCs, the receptive fields are small and distinct and relatively strong simple spike responses were evoked $(+87 \pm 43 \%$; Figures 3C,D) even though the net responses were substantially smaller than in interneurons (Figure 1G). The climbing fiber receptive field corresponds to the inhibitory receptive field in the PC (these two types of receptive fields have a near complete overlap - Ekerot and Jorntell, 2001, 2003 - and the climbing fiber receptive field is therefore not shown separately), and from this skin area the responses evoked by the stimulation was $-48 \pm 18 \%$ relative to the baseline (category VI in Figure 3D, upper graph to the far left). This inhibitory input most likely reflects input from the nearby interneurons, which are activated from the skin area corresponding to the climbing fiber receptive field of the PC (Ekerot and Jorntell, 2001; Jorntell and Ekerot, 2002).

Figures 3B,C illustrate the skin input of a normal Purkinje (left) and after a PF burst stimulation protocol (middle), which expanded the PF receptive field as previously described (Jorntell and Ekerot, 2002). In contrast to PCs with normal PF input, all skin areas now provided input to the cell with inputs exceeding baseline activity by $33-101 \%$. This applied also to the previously inhibitory receptive field ( site \#5 in Figure 3C, category VI in Figure 3D) although we often found that inhibitory and excitatory input could both be evoked from this category of skin area (not shown). In order to induce specific receptive field changes in this state, we applied the PC-skinLTD protocol (which equals the interneuron-skinLTP protocol) using skin site \#3 as the conditioning skin input (Figure 3C, middle; control net response $+47 \pm 22 \%$ ). In contrast to interneurons, this stimulation protocol now resulted in a specific reduction of input (net response $-6 \pm 16 \%$ ) from the stimulated skin site (Figures 3C,D). 


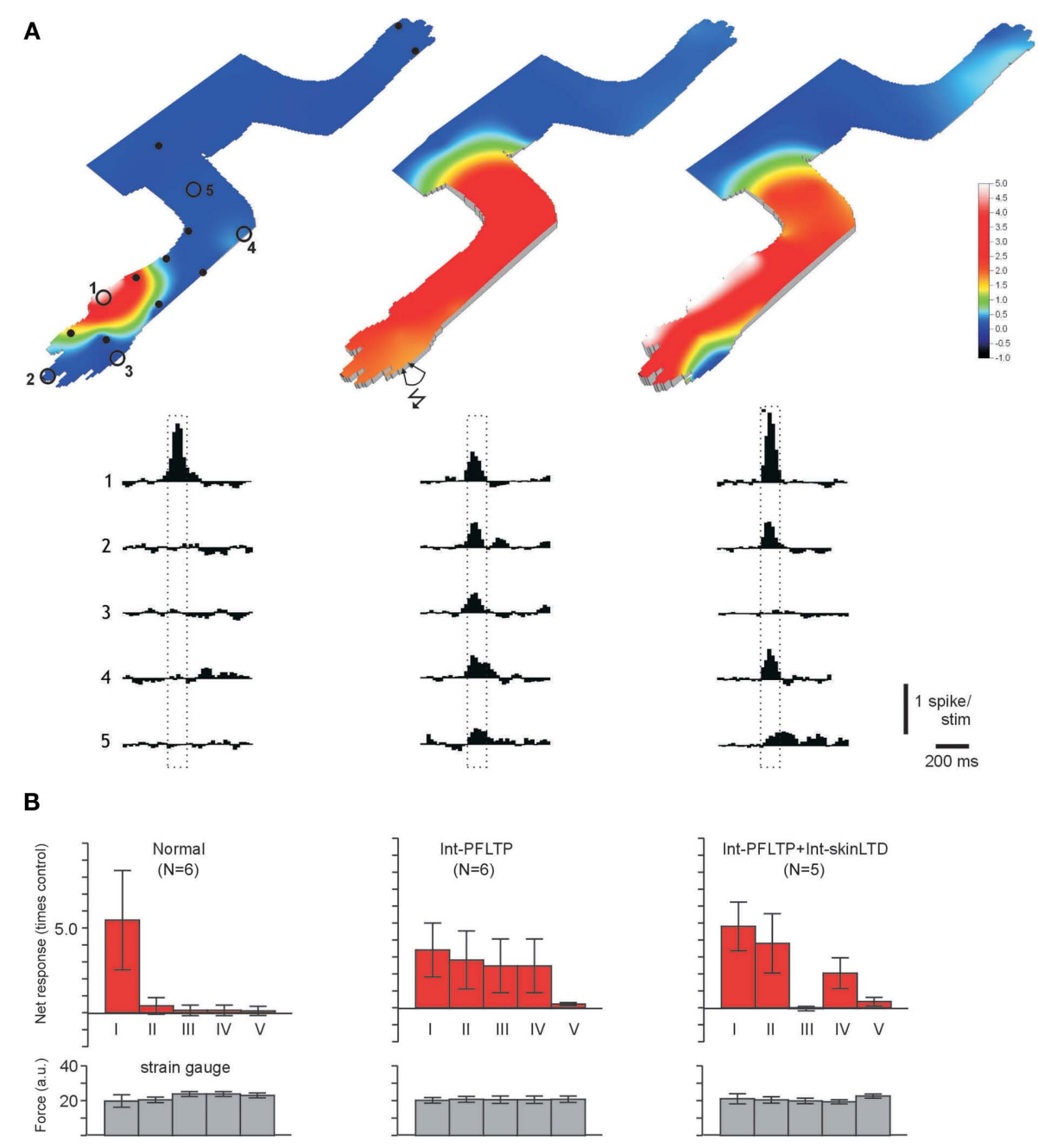

FIGURE 2 | Removal of specific skin inputs in interneurons. (A,B) Display as in Figures 1D-H, but data from another interneuron recorded in its normal state (left), 30 min after an interneuron-PFLTP protocol (middle) and $25 \mathrm{~min}$ after a subsequent (80 min after the interneuron-PFLTP protocol) interneuron-skinLTD protocol (right, the skin burst stimulation was applied to skin site\#3 as illustrated in the middle panel). (B) Quantified input and strain gage signals from different skin sites for normal interneurons, interneurons after interneuron-PFLTP and interneurons after interneuron-PFLTP and interneuron-skinLTD, respectively. Note failure to recruit input from non-forelimb skin areas after the interneuron-PFLTP protocol, in agreement with the findings of Jorntell and Ekerot (2002). Responses were recorded 20-80 min after the respective protocol.
Figure 4 illustrates a similar experiment for a PC that previously had been conditioned by a PC-PFLTP protocol. In this case, two consecutive PC-skinLTD protocols removed two different skin areas from the expanded receptive field, first from the distal forelimb and subsequently from the distal hindlimb. Due to the difficulties in maintaining high quality PC recordings for the exceedingly long time period that was required ( $>4 \mathrm{~h}$ ), this type of experiment, i.e., to remove a second skin are from the simple spike receptive field of the PC, was only tested for one PC, though.

\section{MOSSY FIBER RECEPTIVE FIELDS WERE UNAFFECTED BY SKIN BURST STIMULATION}

The opposite receptive field changes induced in PCs and interneurons by the same stimulation protocol strongly suggested that the induced receptive field changes did not involve changes in the input to mossy fibers (or granule cells). This was also confirmed in direct recordings from mossy fiber terminals (Figure 5A), in which the absence of skin input from the stimulated skin site was confirmed (Figures 5B,C; mossy fiber responses were $+2100 \pm 330 \%$ within the receptive field, compared to $+8 \pm 22 \%$ at the stimulated skin site; $N=8$ ). Mossy fiber recordings were identified as previously described (van Kan et al., 1993; Garwicz et al., 1998).

\section{DISCUSSION}

In the present study, we showed that skin stimulation can be used to add or remove receptive field components in cerebellar cortical neurons. Addition of receptive field components to PCs requires that the skin stimulation is unpaired with CF activation, whereas in interneurons a combined activation of skin bursts and CF is required. The reverse rule applies when receptive field components are to be removed from the two types of neurons. 
A

Ssp
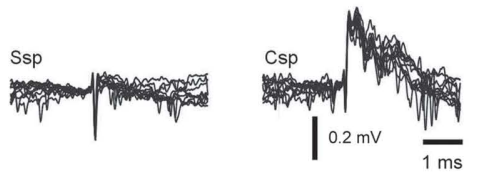

$1_{10 \text { stim }}$

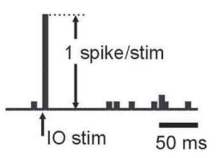

B

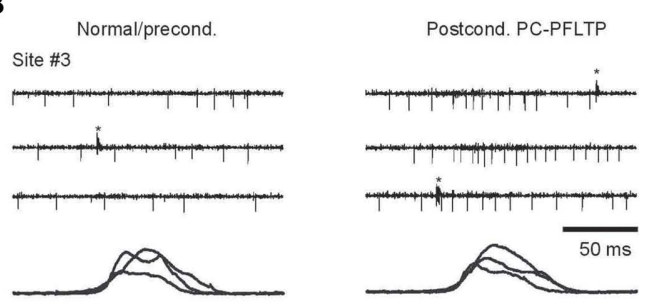

C

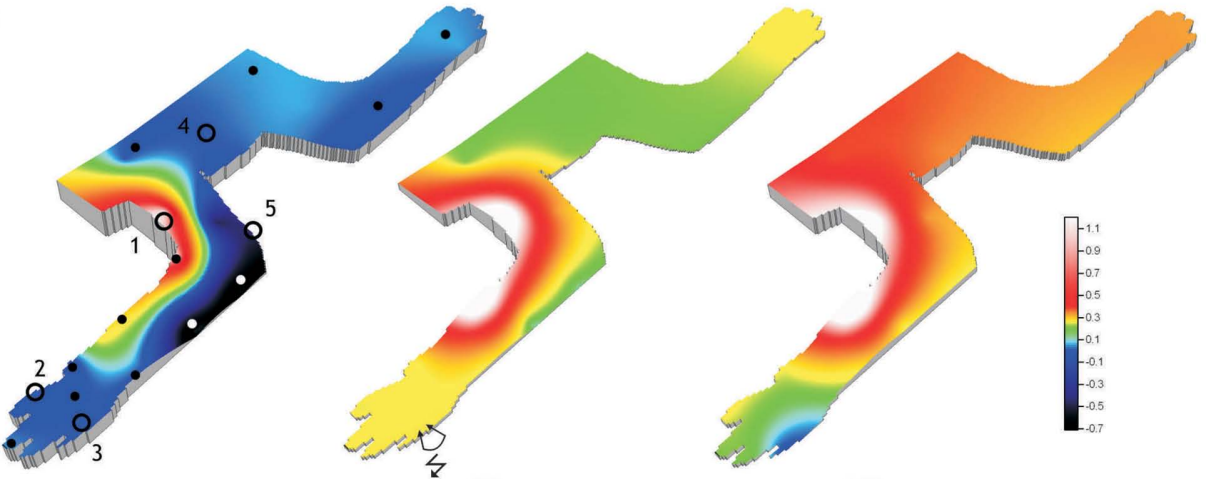

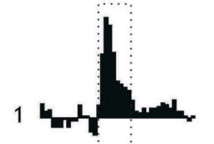
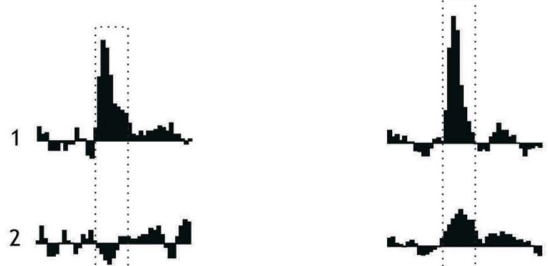

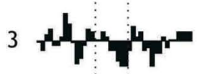
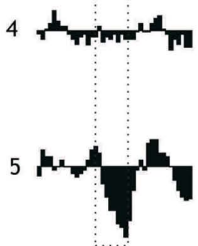

D

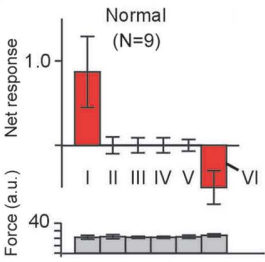

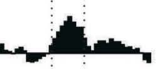
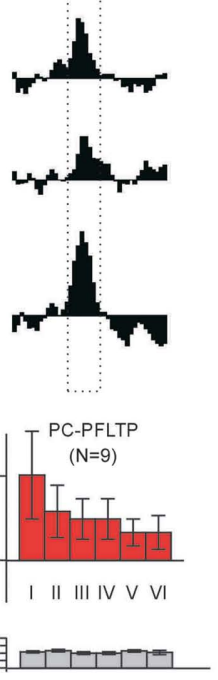
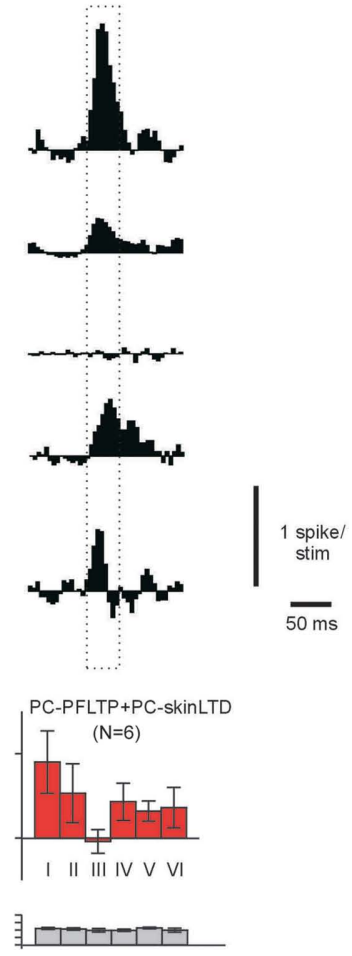

FIGURE 3 | Removal of specific skin inputs in PCs. (A) Superimposed examples of simple spikes (Ssp) and complex spikes (Csp; calibrations to the upper right). The responses of the two spike types to stimulation in the IO $(25 \mu \mathrm{A})$ are summarized in the peristimulus histograms below (calibrations at lower right). Histograms have a bin width of $5 \mathrm{~ms}$. (B) Sample raw traces of spike responses to stimulation of a neutral skin site (\#3, see C) that provided no input in the normal condition. After a protocol for PC-PFLTP, input from that skin site was recruited. Note the strain gage signals below, indicating that a similar amount of stimulation was applied in the two situations. (C) Display as in Figure 2A, but for a PC in the normal state (left), 30 min after a PC-PFLTP protocol (middle) and after a 15 min PC-skinLTD protocol applied to site \#3 (110 min after the PC-PFLTP protocol). As usual in the preconditioned state, the climbing fiber receptive field overlapped the inhibitory receptive field (Ekerot and Jorntell, 2001, 2003), i.e., it was located around the elbow. (D) Display as in Figure 2B. Categories I-V are the same as for the interneurons (category $I$ in the case of PCs corresponding to the simple spike receptive field), but the new category $\mathrm{VI}$ represents the simple spike responses evoked from the climbing fiber (complex spike) receptive field. Responses were recorded 15-80 min after the respective protocol. 


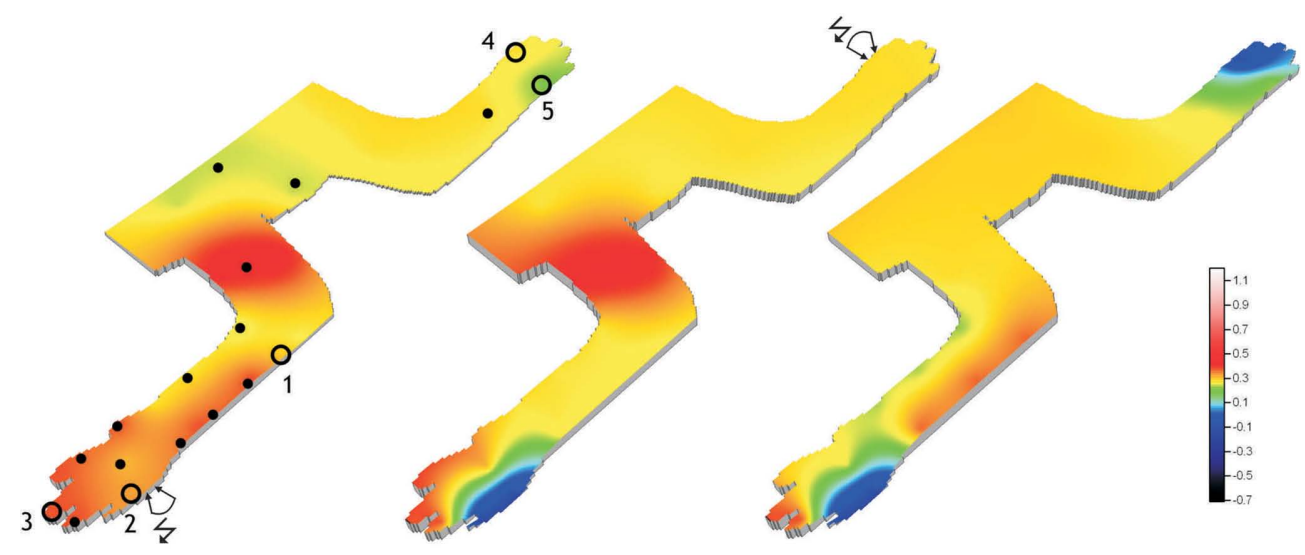

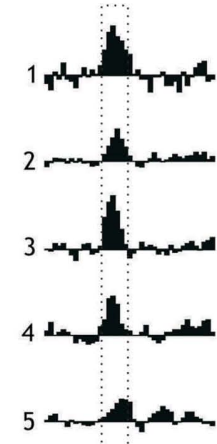

FIGURE 4 | Removal of multiple specific skin input in PCs. Display as in

Figure $\mathbf{3 C}$ for a PC that were conditioned by a PC-PFLTP protocol (left). The climbing fiber receptive field was located on the radial forearm. Middle and right panels

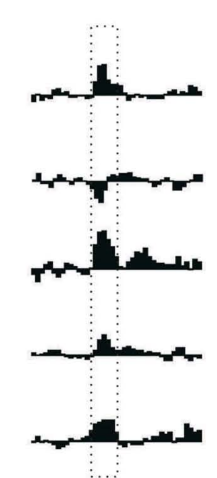

illustrate the changes in input obtained by PC-skinLTD protocols applied in turn to sites \#2 and \#4. They were recorded 20 (120) and 30 (270) min after the PC-skinLTD (number in parenthesis time relative to PC-PFLTP) protocols, respectively.

\section{ELECTRICAL SKIN STIMULATION VERSUS LOCAL FIBER STIMULATION}

How does the electrical skin stimulation used for inducing the specific receptive field changes in the present study relate to natural forms of skin activation that could occur during behavior? Undoubtedly, at the level of the skin, primary afferent spikes would be expected to be elicited in near perfect synchrony, which is unlikely to be a natural pattern of afferent activation. However, primary afferent axons travel a long way to the central nervous system, and widely different conduction velocities in these afferents (Burgess et al., 1968) will serve to desynchronize the incoming volley. In addition, the skin sensory input reaches the mossy fibers of the cerebellum through a variety of specific, parallel ascending pathways, which are characterized by specific conduction delays, synaptic relays and termination areas (Oscarsson, 1973; Jorntell and Ekerot, 2006). In each of these pathways, the temporal pattern of the skin sensory input is specifically transformed due to the network structure and cellular properties in the relays (Bengtsson and Jorntell, 2009). Therefore, both the temporal pattern and, naturally, the spatial pattern of PF synaptic activation (see Introduction) obtained with electrical skin stimulation is much more similar to natural skin activation than what could be achieved through local PF stimulation.
Nevertheless, even though we used repeated manual skin stimulation to the same skin site to map the receptive fields, which theoretically should lead to PC-skinLTP, we never observed any changes in the PC PF receptive fields after receptive field mapping. Presumably, this can be explained by the potentially lower intensity of primary afferent activation obtained from the manual skin stimulation (electrical skin stimulation was delivered as 50 pulses at $333 \mathrm{~Hz}$ /burst) but possibly also by the potentially lower relative synchrony by which the PF synapses were activated. Therefore, the thresholds for inducing these plastic changes by natural skin stimulation would be expected to have higher thresholds, i.e., either require more frequent skin activation than we used, or simply repeated for a longer time.

\section{PLASTICITY PROCESSES UNDERLYING THE RECEPTIVE FIELD CHANGES}

In a previous paper (Jorntell and Ekerot, 2002), in which direct stimulation of PFs was used to induce bidirectional receptive field changes, there was a range of arguments that indicated that the observed changes were due to postsynaptic PF plasticity. The present study could appear somewhat more complicated in terms of the underlying mechanisms since there are many additional synaptic relays that convey the input, i.e., the different mossy fiber pathways (Oscarsson, 1973) and the mossy fiber-granule cell 
A

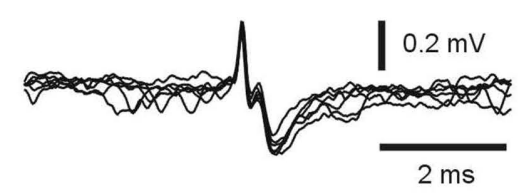

B
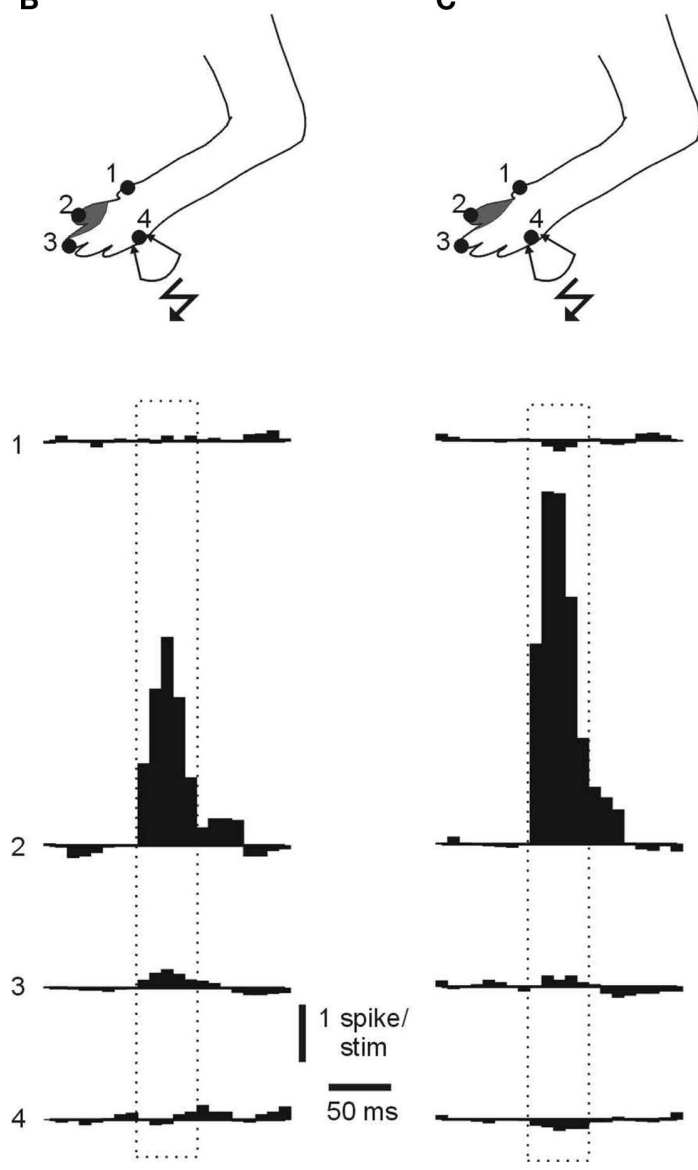

FIGURE 5 | Mossy fiber receptive fields remain normal after skin burst stimulation. (A) Superimposed spikes from a recording from a mossy fiber terminal. Note the very fast spike $(<0.5 \mathrm{~ms})$ and the subsequent "glomerulus potential," which represent the field EPSP in the connected granule cells and Golgi cells. (B) Receptive field, locations of skin sites for which samples of quantified skin input are shown below and stimulation site (\#4) used for skin burst stimulation that resulted in receptive field changes in a PC located nearby. The recording was made about $60 \mathrm{~min}$ after the stimulation protocol. The spontaneous firing rate was $7 \mathrm{~Hz}$ and the evoked spike response was 1990\% higher than the baseline firing. (C) Display as in (B), but for another mossy fiber recorded in the same experiment. The spontaneous firing rate was $12 \mathrm{~Hz}$ and the spike response was $2520 \%$ higher than the baseline firing. Scale bars apply to (B) and (C).

relay. Although we observed that mossy fiber receptive fields were unchanged following skin burst stimulations (Figure 5), the key observations were the reversal of the polarity of the plasticity that depends on the CF and the fact that opposite effects were induced in the two types of neurons by the same protocol. These observations strongly suggest involvement of postsynaptic PF plasticity mechanisms. The possibility that the stimulation protocols could have induced opposite changes in excitability in PCs and interneurons appears to be completely ruled out by the localized receptive field changes obtained.

\section{FUNCTIONAL CONSIDERATIONS}

In the cerebellar $\mathrm{C} 3$ zone, the cutaneous receptive fields of $\mathrm{CF}$, mossy fibers and granule cells have similar locations, distributions and extents (Ekerot et al., 1991; Garwicz et al., 1998; Jorntell and Ekerot, 2006). Based on multiple quantitative analysis methods of their exact location and distribution (Ekerot et al., 1991), these receptive fields have been found to be divisible into a number of distinct subtypes or subclasses, a scheme that was later refined to 40 subclasses (Garwicz et al., 1998). Somewhat surprisingly, we observed that these subtypes of receptive fields appeared to be conserved in the PF input to PCs and interneurons (Ekerot and Jorntell, 2001, 2003). The PF receptive fields in individual PCs and interneurons hence correspond to $2.5 \%$ (1/40) of the available cutaneous PF inputs, and the input from the remaining PF synapses appear to be electrically silent. This estimation correlate well with other investigations in which only 2.6\% (Wang et al., 2000) and $3-7 \%$ (Isope and Barbour, 2002) of the PF synapses on PCs were estimated to be electrically active. From the present study it would seem that input from the 40 different receptive field subtypes can be recruited independently. The subclasses of cutaneous mf-grc inputs could hence be viewed as the information "quanta" of the PF system, which the cerebellar cortex can add or remove to specific PCs and interneurons in order to establish a network that form the input-output associations necessary for smooth interaction with the motor apparatus and the interpositioned neuronal circuitry (reviewed by Dean et al., 2010).

\section{NETWORK CONSIDERATIONS}

Note that the fact that PF inputs corresponding to single subclasses of mossy fiber receptive fields are normally present in PCs and interneurons indicates that there is no convergence of mossy fibers with different cutaneous receptive fields at the granule cell level. This was also recently confirmed by direct observations in intracellular granule cell recordings (Jorntell and Ekerot, 2006; Bengtsson and Jorntell, 2009). In fact, this is not surprising since there is an orderly zonal-microzonal termination of many mossy fiber tracts. Mossy fibers with the same cutaneous receptive fields terminate in longitudinal microzones (Garwicz et al., 1998). In addition to the microzonal organization described for mossy fiber terminals, we have also found that granule cells have a specific depth distribution depending on the type of input they receive (Jorntell and Ekerot, 2006).

These observations argue for a detailed topographical organization within the granule layer that seem to greatly limit the potential for any plasticity in the MF-grc synapses (Gall et al., 2005) to impart any major functional changes to the network, at least in the C3 zone (see also Dean et al., 2010). For example, even if MF plasticity on a granule cell was to result in a switch of one of its presynaptic mossy fibers to another mossy fiber located nearby, chances would be high that the new mossy fiber input would carry basically the same, or at least very nearly related information. This principle can probably be generalized: in systems with a great degree of topographical organization, i.e., in which nerve fibers carrying 
different information have different termination areas, the potential of synaptic plasticity to impart major functional changes is limited. On the contrary, the arrangement of PFs seems to represent a maximal degree of non-topography: also a small population of PFs in a beam contains a plethora of different information. This view is supported by the dramatic receptive field expansions that can be obtained by recruiting input from a small beam of PFs to PCs and interneurons (Figures 2-4; Jorntell and Ekerot, 2002). Here, the potential of any change in synaptic coupling to result in functional change (change in input-output associations) may be at its greatest for the entire central nervous system. For the cerebellar system to be able to compensate for functional changes in the motor apparatus (for example muscle force) and in the interposed neuronal circuitry (for example due to localized vascular insults), PF synaptic plasticity may be required. The cerebellar cortex may be optimally arranged to establish profound changes in wiring on a short time base, and this may indeed be the most important aspect of cerebellar function (Dean et al., 2010).

\section{PLASTICITY IN THE CEREBELLAR AND CEREBRAL CORTICES}

The present results would hence suggest that addition and removal of receptive field components following skin stimulation primarily was the result of LTP and LTD in PF synapses whereas the ascending, afferent mossy fiber and granule cell input is unchanged. In this respect, cerebellar plasticity could be very similar to the model of synaptic changes suggested to explain the receptive field remodeling in the primary visual and sensory cortices (V1 and S1) that follows on lesions blocking parts of the peripheral input. Here, the topology of the thalamocortical (TC) input is strictly confined and considered unable to explain the observed receptive field changes (Darian-Smith and Gilbert, 1995; Qi and Kaas, 2004). Instead, evidence suggests that it is the horizontal cortico-cortical connections that underlie the receptive field changes (Das and Gilbert, 1995; Calford et al., 2003).

Because of their extensive connectivity, the horizontal corticocortical connections and the PFs of the cerebellar cortex are in a prime position to mediate associations. In both the cerebral and cerebellar

\section{REFERENCES}

Apps, R., and Garwicz, M. (2005). Anatomical and physiological foundations of cerebellar information processing. Nat. Rev. Neurosci. 6, 297-311.

Bengtsson, F., and Jorntell, H. (2007). Ketamine and xylazine depress sensory-evoked parallel fiber and climbing fiber responses. J. Neurophysiol. 98, 1697-1705.

Bengtsson, F., and Jorntell, H. (2009). Sensory transmission in cerebellar granule cells relies on similarly coded mossy fiber inputs. Proc. Natl. Acad. Sci. U.S.A. 106, 2389-2394.

Burgess, P. R., Petit, D., and Warren, R. M. (1968). Receptor types in cat hairy skin supplied by myelinated fibers. $J$. Neurophysiol. 31, 833-848.

Calford, M. B., Wright, L. L., Metha, A. B., and Taglianetti, V.(2003). Topographic

cortices, functional subdivisions are to a large extent determined by the topological distribution of incoming and outgoing external connections (Katz and Shatz, 1996; Katz and Crowley, 2002; Apps and Garwicz, 2005). Neocortical columns are defined by the TC input (Mountcastle, 1997) and the cortical connections of many inhibitory interneuron types in run primarily inside the column (Helmstaedter et al., 2009). This forms a very interesting parallel to the cerebellar microzonal/microcomplex structure, which is defined by the distribution of climbing fiber input, and where much of the intracortical inhibitory connections appear to be confined to stay within the microzone (Dean et al., 2010; Jorntell et al., 2010). In both the cerebral and cerebellar cortices, the inputs that defines the functional structure (i.e., TC and climbing fiber inputs, respectively) can be induced to express plastic changes but the inputs nevertheless remains largely confined to within the same functional subdivision (Ichise et al., 2000; Weber et al., 2003; Wimmer et al., 2010). In both types of cortices, it would appear that whereas the functional subdivisions probably are genetically predetermined and hence under normal circumstances very similar between individuals, normal function and information processing requires an experience-driven establishment of associative connections between the functional subdivisions. The mediator of these association-formations would hence seem to be plasticity in the PF connections and in the cortico-cortical connections, respectively. These plasticity processes are presumably most intensely activated under development when the associations are first formed. But, in order to be able to compensate for changes in the afferent signaling or the behavioral consequences of outgoing signaling (for example in disease), it is necessary that these associative connections are plastic throughout adult life.

\section{ACKNOWLEDGMENTS}

The work was supported by SENSOPAC (an Integrated Project funded by the EU under FP6, IST-028056-SENSOPAC), THE (an Integrated Project funded by the EU under FP7, project no. 248587) the Swedish Research Council (project no. K2005-04X14780-03A and K2006-04X-08291-19-3), the Segerfalk Foundation, the Swedish Medical Society.

experimental and computational evidence. Nat. Rev. Neurosci. 11,30-43.

Ekerot, C. F., Garwicz, M., and Schouenborg, J. (1991). Topography and nociceptive receptive fields of climbing fibres projecting to the cerebellar anterior lobe in the cat. $J$. Physiol. (Lond.) 441, 257-274.

Ekerot, C. F., and Jorntell, H. (2001). Parallel fibre receptive fields of Purkinje cells and interneurons are climbing fibre-specific. Eur. J. Neurosci. 13, 1303-1310.

Ekerot, C. F., and Jorntell, H. (2003). Parallel fiber receptive fields: a key to understanding cerebellar operation and learning. Cerebellum 2, 101-109.

Gall, D., Prestori, F., Sola, E., D’Errico, A., Roussel, C., Forti, L., Rossi, P., and D’Angelo, E. (2005). Intracellular calcium regulation by burst discharge determines bidirectional long-term synaptic plasticity at the cerebellum input stage. J. Neurosci. 25, 4813-4822.

Garwicz, M., Jorntell, H., and Ekerot, C. F. (1998). Cutaneous receptive fields and topography of mossy fibres and climbing fibres projecting to cat cerebellar C3 zone. J. Physiol. (Lond.) 512(Pt 1), 277-293.

Helmstaedter, M., Sakmann, B., and Feldmeyer, D. (2009). Neuronal correlates of local, lateral, and translaminar inhibition with reference to cortical columns. Cereb. Cortex 19, 926-937.

Ichise, T., Kano, M., Hashimoto, K., Yanagihara, D., Nakao, K., Shigemoto, R., Katsuki, M., and Aiba, A. (2000). mGluR1 in cerebellar Purkinje cells essential for long-term depression, synapse elimination, and motor coordination. Science 288, 1832-1835.

Isope, P., and Barbour, B. (2002). Properties of unitary granule cell $\rightarrow$ Purkinje cell 
synapses in adult rat cerebellar slices. J. Neurosci. 22, 9668-9678.

Ito, M. (1982). Cerebellar control of the vestibulo-ocular reflex - around the flocculus hypothesis. Annu. Rev. Neurosci. 5, 275-296.

Ito, M. (1984). The Cerebellum and Neural Control. New York: Raven Press.

Ito, M. (2001). Cerebellar long-term depression: characterization, signal transduction, and functional roles. Physiol. Rev. 81, 1143-1195.

Ito, M. (2006). Cerebellar circuitry as a neuronal machine. Prog. Neurobiol. 78, 272-303.

Ito, M., Sakurai, M., and Tongroach, P. (1982). Climbing fibre induced depression of both mossy fibre responsiveness and glutamate sensitivity of cerebellar Purkinje cells. J. Physiol. (Lond.) 324, 113-134.

Jorntell, H., Bengtsson, F., Schonewille, M., and De Zeeuw, C. I. (2010). Cerebellar molecular layer interneurons - computational properties and roles in learning. Trends Neurosci. 33, 524-532.

Jorntell, H., and Ekerot, C. F. (2002). Reciprocal bidirectional plasticity of parallel fiber receptive fields in cerebellar Purkinje cells and their afferent interneurons. Neuron 34, 797-806.

Jorntell, H., and Ekerot, C. F. (2003). Receptive field plasticity profoundly alters the cutaneous parallel fiber synaptic input to cerebellar interneurons in vivo. J. Neurosci. 23, 9620-9631.
Jorntell, H., and Ekerot, C. F. (2006). Properties of somatosensory synaptic integration in cerebellar granule cells in vivo. J. Neurosci. 26, 11786-11797.

Jorntell, H., and Hansel, C. (2006). Synaptic memories upside down: bidirectional plasticity at cerebellar parallel fiber-Purkinje cell synapses. Neuron 52, 227-238.

Katz, L. C., and Crowley, J. C. (2002). Development of cortical circuits: lessons from ocular dominance columns. Nat. Rev. Neurosci. 3, 34-42.

Katz, L. C., and Shatz, C. J. (1996). Synaptic activity and the construction of cortical circuits. Science 274, 1133-1138.

Lev-Ram, V., Mehta, S. B., Kleinfeld, D., and Tsien, R.Y. (2003). Reversing cerebellar long-term depression. Proc. Natl. Acad. Sci. U.S.A. 100, 15989-15993.

Lev-Ram, V., Wong, S. T., Storm, D. R., and Tsien, R. Y. (2002). A new form of cerebellar long-term potentiation is postsynaptic and depends on nitric oxide but not cAMP. Proc. Natl. Acad. Sci. U.S.A. 99, 8389-8393.

Malenka, R. C., and Bear, M. F. (2004). LTP and LTD: an embarrassment of riches. Neuron 44, 5-21.

Miyakawa, H., Lev-Ram, V., Lasser-Ross, N., and Ross, W. N. (1992). Calcium transients evoked by climbing fiber and parallel fiber synaptic inputs in guinea pig cerebellar Purkinje neurons. J. Neurophysiol. 68, 1178-1189.

Mountcastle, V. B. (1997). The columnar organization of the neocortex. Brain 120(Pt 4), 701-722.
Niedermayer, E., and Lopes da Silva, F. (1993). Electroencephalography: Basic Principles, Clinical Applications, and Related Fields. Baltimore: Williams and Wilkins.

Oscarsson, O. (1973). "Functional organization of spinocerebellar paths," in Handbook of Sensory Physiology, Vol. II: Sensory System, ed. A. Iggo (New York: Springer Verlag), 339-380.

Qi, H. X., and Kaas, J. H. (2004). Myelin stains reveal an anatomical framework for the representation of the digits in somatosensory area $3 \mathrm{~b}$ of macaque monkeys. J. Comp. Neurol. 477, 172-187.

Rancillac, A., and Crepel, F. (2004) Synapses between parallel fibres and stellate cells express long-term changes in synaptic efficacy in rat cerebellum. J. Physiol. 554, 707-720.

Smith, S.L., and Otis, T. S. (2005). Patterndependent, simultaneous plasticity differentially transforms the inputoutput relationship of a feedforward circuit. Proc. Natl. Acad. Sci. U.S.A. 102, 14901-14906.

Szapiro, G., and Barbour, B. (2007) Multiple climbing fibers signal to molecular layer interneurons exclusively via glutamate spillover. Nat. Neurosci. 10, 735-742.

van Kan, P. L., Gibson, A. R., and Houk, J. C. (1993). Movement-related inputs to intermediate cerebellum of the monkey. J. Neurophysiol. 69, 74-94.

Wang, S. S., Khiroug, L., and Augustine, G. J. (2000). Quantification of spread of cerebellar long-term depression with chemical two-photon uncaging of glutamate. Proc. Natl. Acad. Sci. U.S.A. 97, 8635-8640.

Weber, J. T., De Zeeuw, C. I., Linden, D. J., and Hansel, C. (2003). Long-term depression of climbing fiber-evoked calcium transients in Purkinje cell dendrites. Proc. Natl. Acad. Sci. U.S.A. 100, 2878-2883.

Wimmer, V. C., Broser, P. J., Kuner, T., and Bruno, R. M. (2010). Experienceinduced plasticity of thalamocortical axons in both juveniles and adults J. Comp. Neurol. 518, 4629-4648.

Conflict of Interest Statement: The authors declare that the research was conducted in the absence of any commercial or financial relationships that could be construed as a potential conflict of interest.

Received: 10 November 2010; accepted: 18 February 2011; published online: 03 March 2011.

Citation: Jörntell H and Ekerot C-F (2011) Receptive field remodeling induced by skin stimulation in cerebellar neurons in vivo. Front. Neural Circuits 5:3. doi: 10.3389/ fncir.2011.00003

Copyright (c) 2011 Jörntell and Ekerot.

This is an open-access article subject to an exclusive license agreement between the authors and Frontiers Media SA, which permits unrestricted use, distribution, and reproduction in any medium, provided the original authors and source are credited. 